\title{
Isolation and Characterization of Canine Amniotic Membrane-Derived Multipotent Stem Cells
}

\author{
Sang-Bum Park ${ }^{1,2,39}$, Min-Soo Seo ${ }^{1,2,39}$, Hyung-Sik Kim ${ }^{1,2,3}$, Kyung-Sun Kang ${ }^{1,2,3 *}$ \\ 1 Adult Stem Cell Research Center, College of Veterinary Medicine, Seoul National University, Seoul, Republic of Korea, 2 Laboratory of Stem Cell and Tumor Biology, \\ Department of Veterinary Public Health, College of Veterinary Medicine, Seoul National University, Seoul, Republic of Korea, 3 BK 21 Program for Veterinary Sciences, \\ College of Veterinary Medicine, Seoul National University, Seoul, Republic of Korea
}

\begin{abstract}
Recent studies have shown that amniotic membrane tissue is a rich source of stem cells in humans. In clinical applications, the amniotic membrane tissue had therapeutic effects on wound healing and corneal surface reconstruction. Here, we successfully isolated and identified multipotent stem cells (MSCs) from canine amniotic membrane tissue. We cultured the canine amniotic membrane-derived multipotent stem cells (CAM-MSCs) in low glucose DMEM medium. CAM-MSCs have a fibroblast-like shape and adhere to tissue culture plastic. We characterized the immunophenotype of cAM-MSCs by flow cytometry and measured cell proliferation by the cumulative population doubling level (CPDL). We performed differentiation studies for the detection of trilineage multipotent ability, under the appropriate culture conditions. Taken together, our results show that CAM-MSCs could be a rich source of stem cells in dogs. Furthermore, cAM-MSCs may be useful as a cell therapy application for veterinary regenerative medicine.
\end{abstract}

Citation: Park S-B, Seo M-S, Kim H-S, Kang K-S (2012) Isolation and Characterization of Canine Amniotic Membrane-Derived Multipotent Stem Cells. PLoS ONE 7(9): e44693. doi:10.1371/journal.pone.0044693

Editor: Joseph Alan Bauer, Bauer Research Foundation, United States of America

Received April 25, 2011; Accepted August 10, 2012; Published September 14, 2012

Copyright: (C) 2012 Kang et al. This is an open-access article distributed under the terms of the Creative Commons Attribution License, which permits unrestricted use, distribution, and reproduction in any medium, provided the original author and source are credited.

Funding: This work was supported by a National Research Foundation of Korea (NRF) grant funded by the Korean government (MEST, 2010-0020265). The funders had no role in study design, data collection and analysis, decision to publish, or preparation of the manuscript.

Competing Interests: The authors have declared that no competing interests exist.

*E-mail: kangpub@snu.ac.kr

9 These authors contributed equally to this work.

\section{Introduction}

Recently, the study of stem cells has focused on the therapeutic effects of the cells. Stem cells have a multipotent differentiation capacity and self-renewal ability. Initially, multipotent stem cells (MSCs) were isolated and characterized from bone marrow [1]. The current sources of MSCs are from various tissues such as umbilical cord blood, adipose tissue, amniotic fluid and peripheral blood [2-6]. The placenta has an important role in the development and survival of the fetus, supplying nutrients and oxygen. The placenta has three-layer structure including the amnion, chorion and deciduas [7]. Commonly, after parturition, the placenta is treated as medical waste and discarded. However, amniotic membranes have the potential to be used as a clinical application for wound healing and cornea surface reconstruction [7]. In humans, there are reports of MSCs derived from placenta, especially, from the amniotic membrane $[8,9]$.

Additionally, we demonstrated that cAM-MSCs have a multilineage differentiation capacity and self-renewal ability. Our results showed that the typical morphology of cAM-MSCs is similar to that of human MSCs and that, the cells displayed vigorous cell proliferation. In the differentiation studies, cAMMSCs showed adipogenesis, osteogenesis, neurogenesis and chondrogenesis ability in vitro. Therefore, we suggest that cAMMSCs may represent a rich source of stem cells that may be useful in veterinary medicine. Furthermore, cAM-MSCs could be used therapeutically in canine regenerative medicine studies.

\section{Materials and Methods}

Although we use animal tissue, harvesting of tissues did not involve any invasive or inhumane methodology. In fact, sacrificing live animal for harvesting was not needed at all. We used amniotic membranes that are normally discarded after they are separated by cesarean sectioning for deliveries in our department (Seoul National University Hospital for Animal). The cesarean sections were carried out for non-research purpose at approximately 60-69 days of gestation by qualified veterinarians. They were provided free of charge by the department for the purposes of the study. Such isolated membranes were only used in isolating and characterizing stem cells from the tissue. All safety compliances were strictly observed and adhered to the Policy and Regulation for Care and Use of Laboratory Animals (Institute of Laboratory Animal Resources Seoul National University).

\section{Animals}

Healthy adult mixed-breed dogs $(\mathrm{n}=6 ; 4.5 \pm 0.4 \mathrm{~kg})$ were used. Applicable institutional and governmental regulations concerning the ethical use of animals were followed during the course of this research. This investigation was performed in accordance with the guidelines of the "Guide for the Care and Use of Laboratory Animals" of Seoul National University. In the cesarean-section delivery, the dogs were pre-medicated with acepromazine maleate (0.1 mg/kg; Sedaject, Samwoo medical, Yesan, Korea) and then, thiopental sodium (15 mg/kg; Pentotal, Joongwei pharmaceutical, Seoul, Korea) was injected intravenously to induce anesthesia. 
Table 1. List of PCR primers for differentiation markers.

\begin{tabular}{|c|c|c|c|c|c|}
\hline Markers & Name & Sequence of primers & Size & Cycle & Temp. $\left({ }^{\circ} \mathrm{C}\right)$ \\
\hline & LPL & Forward : ACACATTCACAAGAGGGTCAC Reverse : CTCTGCAATCACACGGATG & 132 & 32 & 60 \\
\hline \multirow[t]{3}{*}{ Adipocyte } & LEPTIN & $\begin{array}{l}\text { Forward : CTATCTGTCCTGTGTTGAAGCTG Reverse : } \\
\text { TGTGTGAAATGTCATTGATCCTG }\end{array}$ & 102 & 32 & 60 \\
\hline & FABP4 & Forward : ATCAGTGTAAACGGGGATGTG Reverse : GACTITTCTGTCATCCGCAGTA & A117 & 32 & 60 \\
\hline & SPARC & Forward : TGAGAAGGTATGCAGCAACG Reverse : AGTCCAGGTGGAGTTGTGG & 110 & 32 & 56 \\
\hline \multirow[t]{3}{*}{ Osteocyte } & MSX2 & Forward : TCCGCCAGAAACAATACCTC Reverse : AAGGGTAGGACGCTCCGTAT & 243 & 32 & 56 \\
\hline & COL1A1 & Forward : CACCTCAGGAGAAGGCTCAC Reverse : ATGTTCTCGATCTGCTGGCT & 124 & 32 & 56 \\
\hline & BGLAP & Forward : GTGGTGCAACCTTCGTGTC Reverse : GCTCGCATACTTCCCTCTTG & 132 & 34 & 58 \\
\hline \multirow[t]{2}{*}{ Neurocyte } & GFAP & Forward : TCCGAGGGGGCAAAAGCACC Reverse : GGCAGGCTGCTAACCGAGAGC & C104 & 30 & 62 \\
\hline & MAP2 & Forward : CAGCGACAAGGCCGACACGT Reverse : GGGCCAAACTCGACACCCGG & 336 & 34 & 66 \\
\hline \multirow[t]{2}{*}{ Chondrocyte } & COL2A1 & Forward : GAAACTCTGCCACCCTGAATG Reverse : GCTCCACCAGTTCTTCTTGG & 156 & 34 & 64 \\
\hline & AGGRECAN & $\begin{array}{l}\text { Forward : ATCAACAGTGCTTACCAAGACA Reverse : } \\
\text { ATAACCTCACAGCGATAGATCC }\end{array}$ & 122 & 32 & 60 \\
\hline Housekeeping & GAPDH & Forward : AACATCATCCCTGCTTCCAC Reverse : TCCTTGGAGGCCATGTAGAC & 392 & 24 & 58 \\
\hline
\end{tabular}

Isoflurane (AErrane, Baxter, Mississauga, ON, Canada) was used to maintain anethesia. Under sterile conditions, the procedure was performed.

\section{Cell Isolation and Culture}

Cell isolation was performed as previously described with some modification $[8,10]$. All placental samples were obtained through cesarean-section deliveries from canines. To separate the amniotic membrane from the whole placenta, the amniotic membrane was peeled off from the chorionic membrane mechanically. Under sterile conditions, the collected amniotic membranes were rinsed with normal saline $(0.9 \%)$ several times. Generally, amniotic membrane (AM) composed of epithelial monolayer and an avascular stroma. The collected amniotic membranes were conducted with enzymatic and mechanical digestion treatment in 2 steps. Step 1; to avoid contamination of epithelial cells, in cell isolation of amniotic membrane, the collected AM was treated with trypsin-EDTA $(0.25 \%)$ at $37^{\circ} \mathrm{C}$ for 30 minutes. After trypsinEDTA treatment, the AM was washed with normal saline for $3 \sim 4$ times. Step 2; to isolate pure mesenchymal cells from canine AM without epithelial cells, the washed AM was chopped with a surgical blade. Thereafter, it was digested in collagenase type I ( $2 \mathrm{mg} / \mathrm{ml}$; Worthington biochemical, Freehold, NJ) at $37^{\circ} \mathrm{C}$ for approximately 3 4 hours. After enzyme digestion, washed in phosphate-buffered saline (PBS) (Cellgro, USA) by centrifugation at $350 \times g$ for $5 \mathrm{~min}$. The cell pellet was resuspended in the basal culture medium, low glucose Dulbecco's Modified Eagle's Medium (LG-DMEM; Gibco BRL, USA) containing 10\% FBS (Fetal bovine serum; Gibco BRL, USA). The cells were seeded into T75 polystyrene cell culture flasks (Nunc, USA) and incubated in a humidified atmosphere with $5 \% \mathrm{CO}_{2}$. The basal culture medium was changed 3 times a week and passaged once the cells reached $80-90 \%$ confluency.

\section{Cumulative Population Doubling Level Analysis}

The proliferation and growth efficiency of cAM-MSCs were determined by the total cumulative population doubling level (CPDL) using the formula CPDL $=\ln (\mathrm{Nf} / \mathrm{Ni}) \ln 2$, where $\mathrm{Ni}$ is the initial seeding cell number, $\mathrm{Nf}$ is the final harvest cell numbers and $\ln$ is the natural $\log$. The cells $\left(5 \times 10^{4}\right)$ were plated in triplicate in a 6-well culture plate (Nunc) and subcultured 5-7 days later. The final cell numbers were counted and $5 \times 10^{4}$ cells were replated. To yield the cumulated doubling level, the population doubling for each passage was calculated and then added to the population doubling levels of the previous passages.

\section{Flow Cytometry}

To determine the immunophenotype of cAM-MSGs, the cells were stained with specific antibodies for FACS analysis, following the protocol provided by the supplier (BD Biosciences, USA). Briefly, the cAM-MSGs were trypsinized and washed several times with PBS. The suspended cells were aliquoted (approximately $1 \times 10^{6}$ cells) for specific antibody staining. The cells were immunostained with the following antibodies: Mouse anti-human CD3, mouse anti-human CD1lc, mouse anti-human CD28, mouse anti-human CD34, mouse anti-human CD38, mouse antihuman CD4la, mouse anti-human CD45, mouse anti-human CD62L, mouse anti-human CD90 (BD Biosciences) and mouse anti human CD105 (Serotec, USA). The antibodies were conjugated with Fluorescein isothiocyanate (FITC) or phycoerythrin (PE). Analysis was determined by the use of FACS Calibur $^{\text {TM }}$ (BD Biosciences) and Cell Quest Pro ${ }^{\text {TM }}$ (BD Biosciences) software.

\section{Adipogenesis}

To determine if the cAM-MSCs could differentiate into adipocytes the cells were treated with adipogenic differentiation medium containing dexamethasone $(1 \mathrm{uM})$, indomethacin (60 uM), 3-isobutyl-1-metyl-xanthine (500 uM; IBMX) and insulin ( $5 \mathrm{ug} / \mathrm{ml}$ ) (Sigma-Aldrich, USA). The cells were treated with the adipogenic differentiation medium for 3 weeks at passage 5 once they had reached 80 90\% confluency. After 3 weeks, Oil Red O staining was performed to detect lipid droplets. The cells were fixed with $10 \%$ formalin for fixation at least 1 hour and rinsed with $60 \%$ isopropanol prior to incubation in freshly diluted Oil Red O for 10 minutes. Stains were solubilized using 100\% isopropanol, and the resulting absorbande was measured at $500 \mathrm{~nm}$ using a spectrophotometer [11]. 
Table 2. List of quatitative RT-PCR primers for stem cell markers.

\begin{tabular}{lll}
\hline & & \\
\hline Name & Sequence of primers & Temp. $\left({ }^{\circ}\right.$ C) \\
\hline OCT4 & Forward : TCGTGAAGCCGGACAAGGAGAAG Reverse : AGGAACATGTTCTCCAGGTTGCCT & 60 \\
SOX2 & Forward : AACCCCAAGATGCACAACTC Reverse : CGGGGCCGGATTATAATC & 60 \\
NANOG & Forward : CCTGCATCCTTGCCAATGTC Reverse : TCCGGGCTGTCCTGAGTAAG & 60 \\
KLF4 & Forward : CCATGGGCCAAACTACCCAC Reverse : TGGGGTCAACACCATTCCGT & 60 \\
\hline doi:10.1371/journal.pone.0044693.t002 &
\end{tabular}

\section{Osteogenesis}

Osteogenic differentiation medium containing ascorbic acid 2phosphate $(50 \mu \mathrm{M})$, dexamethasone $(100 \mathrm{nM}), \beta$-glycerophosphate $(10 \mathrm{mM})$ (Sigma-Aldrich, USA) and 10\% FBS in LGDMEM was used to determine osteogenic differentiation capability. When the cells reached at $80 \sim 90 \%$ confluency, the medium was changed to the osteogenic differentiation medium, and the cells incubated for a further 3 weeks at passage 5. After 3 weeks, the calcium deposition was detected by staining with Alizarin Red S. The cells were washed with PBS and, fixation was performed with ice-cold ethanol $(70 \%)$ for 1 hour at $4^{\circ} \mathrm{C}$. The cells were then washed several times with distilled water. The cells were stained with Alizarin Red S (40 mM; pH 4.2; Sigma-Aldrich, USA) for $10 \mathrm{~min}$ at room temperature. The cells were rinsed of non-specific dye with five washes of distilled water. Alizarin Red S staining was solubilized using cetylpyridinium chloride (100 mM; Sigma-Aldrich, USA) for 1 hour. Solubilized Alizarin Red S absorbance was measured at $570 \mathrm{~nm}$ using a spectrophotometer [11].

\section{Neurogenesis}

Neural differentiation medium was used to induce neurogenesis. The cells were seeded at passage 5 in the basal culture medium and allowed to reach confluency. To induce differentiation, the cells were incubated with Beta-mercaptoethanol (1 mM; BME Sigma-Aldrich, USA) and 5\% FBS for 24 hours prior to induction. Following this, the cells were treated with serum-free induction medium containing Docosahexaenoic (100 $\mu \mathrm{M}$; DHA, Sigma-Aldrich, USA), B27 supplement (Gibco BRL, USA) and $1.5 \%$ Dimethyl sulfoxide (DMSO, SigmaAldrich, USA for 2 days [12].

\section{Chondrogenesis}

To promote the cAM-MSGs differentiate into chondrocytes, chondrogenic differentiation medium was used. The cells $\left(5 \times 10^{5}\right)$ were seeded in a $15 \mathrm{ml}$ polypropylene tube and centrifuged to a pellet at passage 5 . The pellets were cultured in $1 \mathrm{ml}$ of chondrogenic differentiation medium (Lonza) and incubated for 3 weeks. The medium was changed 3 times a week. After differentiation, the pellets were embedded in paraffin and $3 \mu \mathrm{m}$ sections were cut. To detect chondrogenesis, the sections were stained with toluidine blue following standard protocols [13].

\section{Immunostaining}

Mouse anti-neural specific beta III tubulin (Abcam, UK) and rabbit anti-Glial Fibrillary Acidic Protein (GFAP, Millipore, USA) antibodies were used for immunostaining. Cells were fixed with $4 \%$ paraformaldehyde for $20 \mathrm{~min}$, and were then permeabilized in $0.5 \%$ Triton-X 100 at room temperature for $10 \mathrm{~min}$. After washing with PBS several times, the cells were blocked with $10 \%$ normal goat serum (NGS) overnight at $4^{\circ} \mathrm{C}$. Primary antibodies were incubated for 2 hours at room temperature. After PBS washing, the cells were incubated with secondary antibodies Alexa 488 \& 594 (1:1000, Molecular Probe, Inc., Eugene, OR, USA) for 1 hour. Finally, for nuclear staining, the samples were incubated for 15 minutes with Hoechst $33238(1 \mathrm{mg} / \mathrm{ml})$, diluted 1:100 in PBS. Images were captured using a confocal microscope (Eclipse TE2000; Nikon, Japan).

\section{RT-PCR}

Total RNA was extracted from the cultured cells with the Easyspin total RNA extraction kit (Intron Biotechnology, Seongnam, Korea) according to the manufacturer's protocol. RNA concentrations were measured by absorbance at $260 \mathrm{~nm}$ with a spectrophotometer. cDNA was prepared by $1 \mu \mathrm{g}$ of total RNA for reverse transcription using Superscript II reverse transcriptase (Invitrogen, Carlsbad, CA) and oligo (dT) primers (Invitrogen). The cDNA was amplified by PCR using Platinum Taq (Invitrogen, Carlsbad, CA). The PCR primers are shown in the Table 1. The PCR products were separated on a $1.5 \%$ agarose gel and visualized with ethidium bromide.

\section{Quantitative RT-PCR}

Quantitative RT-PCR was performed by mixing cDNA with primers and Power SYBR Green PCR Master Mix (Applied Biosystems, Foster City, CA). Quantitative RT-PCR was performed using an ABI 7500 Realtime-PCR System with supplied software (Applied Biosystems), according to the manufacturer's instructions. RNA expression levels were compared after normalization to endogenous glyceraldehyde-3-phosphate dehydrogenase (GAPDH). The primer sequences used in this study are listed in Table 1 \& 2.

\section{Results}

\section{Primary Culture of CAM-MSCs}

Placental tissues were collected from canine cesarean-section deliveries. We mechanically peeled off the amniotic membrane tissues from the placental tissue (Figure 1A). We isolated and cultured the primary stem cells from the canine amniotic membrane tissue. The cAM-MSGs displayed the spindle-shaped morphology typical of MSGs, and were adherent to the plastic culture surface (Figure 1B). For cell proliferation assays, we measured and calculated the cell population via CPDL. The cells were seeded $\left(5 \times 10^{4}\right.$ cells/well $)$ in a 6 -well culture plate and subcultured 5-7 days later. This was repeated until we observed a decrease in the proliferation rate, from passage 3 to 20. The curve of the rate of growth steadily increased through the cumulative population was observed (Figure 1C).

\section{Expression Pattern of Stem Cell Markers}

To measure gene expression levels of stem cell markers, quantitative RT-PCR was performed in passage dependant 

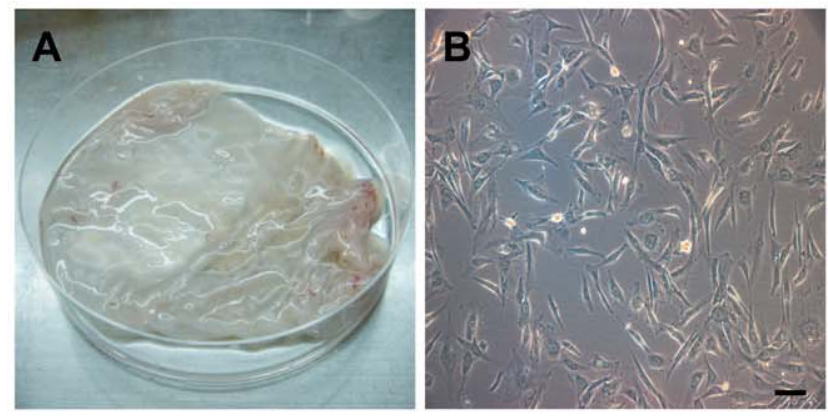

C
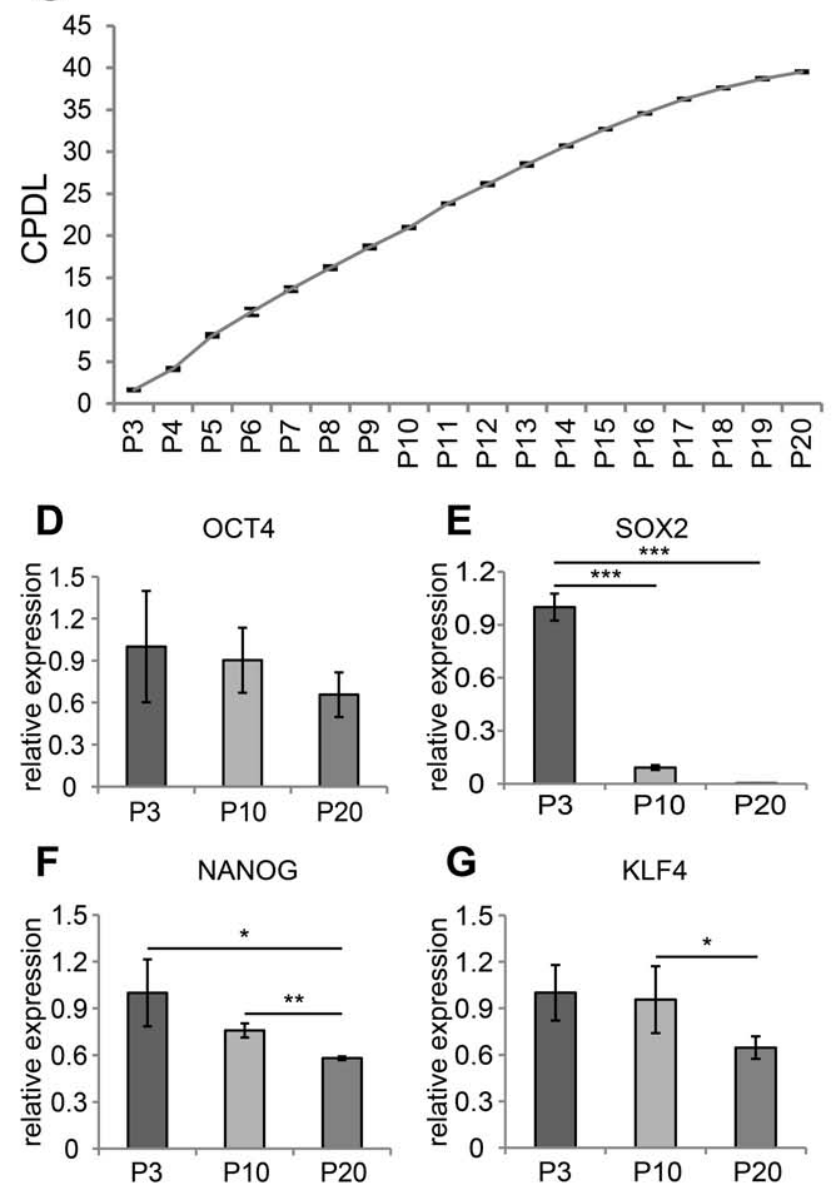

Figure 1. Primary culture of CAM-MSCs and the evaluation of CPDL \& stem cell markers. (A) Harvesting of canine amniotic membrane tissue. (B) Phase contrast images of CAM-MSCs. Cells were cultured with DMEM (10\% FBS). Cultured cells exhibited a fibroblast-like morphology and spindle shape similar to that displayed by human mesenchymal stem cells. Scale bar $=50 \mu \mathrm{m}$. (C) Measuring CPDL of CAM-MSCs. CPDL was evaluated with the formula described in the Materials and Methods section. The CPDL was measured from passage 3 to 20. Cells grew consistently until passage 20. (D-G) Quantitative RTPCR assay for evaluation of stem cell markers: OCT4, SOX2, NANOG and KLF4. We performed all these analyses in triplicate and the mean $+/-$ the standard deviation plotted $\left({ }^{*} ; \mathrm{p}<0.5,{ }^{* *} ; \mathrm{p}<0.01,{ }^{* * *} ; \mathrm{p}<0.001\right)$. doi:10.1371/journal.pone.0044693.g001

manner (passage 3,10 and 20). The stem cell markers, such as OCT4, SOX2, NANOG and KLF4, showed the decreasing expression pattern as a function of increased passages (Figurel D$\mathrm{G})$.

\section{Immunophenotype of cAM-MSCs}

To identify the immunophenotype of cAM-MSCs, cell surface specific markers were examined via FACS analysis. Commonly, MSCs show definite specific cell surface markers. According to the International Society of Cellular Therapy, MSCs have positive expression of CD90 and CD105, but negative expression of CD11c, CD34 and CD45 surface antigens [14]. For FACS analysis of cAM-MSCs, we used 10 CD markers (CD3, CD11c, CD28, CD34, CD38, CD41a, CD45, CD62L, CD90 and CD105) to distinguish the MSC phenotype. For FACS analysis showed that, cAM-MSGs have an expression pattern consistent with the MSC immunophenotype (Figure 2). The results showed that cAMMSCs showed positive expression of CD90 and CD105, which are well-known, typical MSCs markers. CD90 is called Thy-1 and is a marker for various types of stem cells, such as endometrial stem cells, hepatic stem cells, keratinocyte stem cells and mesenchymal stem cells [15-17]. CD105 is also called SH2 and is a well-known MSC marker [14]. However, the cells were negative for the expression of other immune cells markers (CD3, GD11c, GD28, CD38 and CD62L), hematopoietic cells (CD34, CD45) and markers of platelets (CD4la). These results show that the immunophenotype of the cAM-MSCs is consistent with that of other characterized MSCs.

\section{Adipogenesis}

To confirm their adipogenic differentiation ability, cAM-MSCs were treated with adipogenic induction medium for 3 weeks. After differentiation, the cells were stained with Oil Red $\mathrm{O}$ for the detection of fatty droplets. The basal culture medium was used as a control condition. We detected the fatty droplets under differentiation conditions, but not under control conditions (Figure 3A-D). To quantify the differentiation status of the cells, the Oil Red O was eluted with $100 \%$ isopropanol and the absorbance measured (Figure 3E). The differentiated cells displayed absorbance values that were 5 -fold greater than those of the control cells. Additionally, we measured the gene expression levels of markers associated with adipogenesis, such as FABP4, Leptin and LPL via RT-PCR (Figure 3F) and quantitative RTPCR (Figure 3G). After differentiation, the adipogenic-associated markers were increased in treated cells compared to controls (Figure 3F, G).

\section{Osteogenesis}

To confirm their osteogenic differentiation ability, cAM-MSCs were treated with osteogenic induction medium for 3 weeks. Alizarin Red S staining, which positively stains calcium depositions, was used to detect differentiation. Basal culture medium was used as a control condition. Under differentiation conditions, there was strong, positive Alizarin Red S staining. Negative staining was observed under control conditions (Figure 4A-D). To quantify the differentiation status, stain from all cells was eluted with $100 \mathrm{mM}$ of cetylpyridinium chloride and absorbance measured. The differentiated cells displayed about 15-fold greater values than control cells. Additionally, we measured the gene expression levels of markers associated with osteogenesis, such as MSX2, SPARC, COL1A1 and BGLAP via RT-PCR (Figure 4F) and quantitative RT-PGR (Figure 4G). After differentiation, the osteogenic associated markers were increased compared to controls (Figure 4F, G).

\section{Neurogenesis}

To confirm their differentiation ability, cAM-MSCs were treated with neural differentiation medium. After differentiation, 

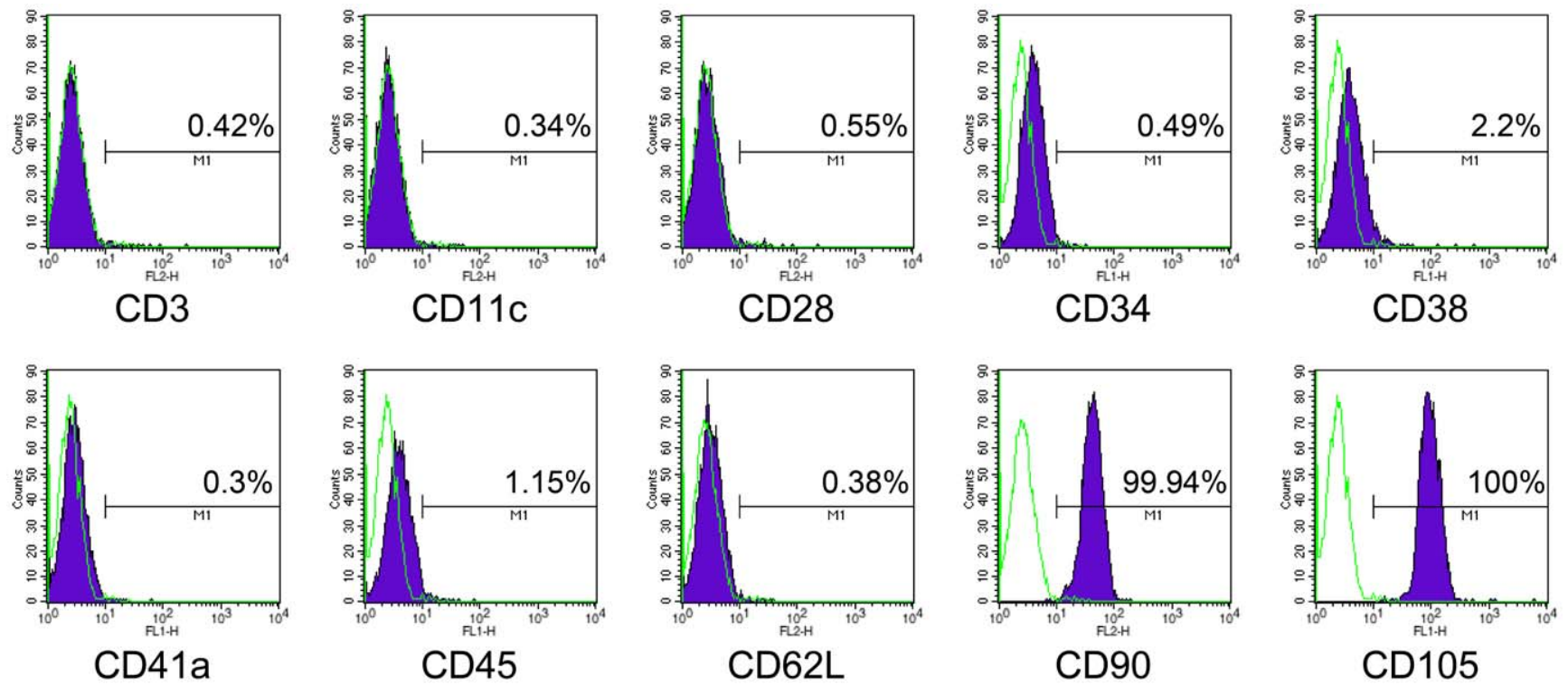

Figure 2. FACS analysis of CAM-MSCs. Analysis was performed at passage 5 . Values show the intensity of the indicated antigen. doi:10.1371/journal.pone.0044693.g002

we performed immunostaining and RT-PCR. Basal culture medium was used as a control condition. By immunostaining, we showed that neural markers GFAP and beta III tubulin were positively expressed under differentiation conditions (Figure 5C, D). However, under the basal culture condition, cAM-MSCs expressed GFAP but, not beta III tubulin (Figure 5A, B). The negative control was incubated with secondary antibodies Alexa 488 \& 594 demonstrated no background signal (Figure 5E-5F). When we measured the expression levels of neural-associated genes via RT-PCR (Figure $5 \mathrm{H}$ ) and quantitative RT-PCR (Figure 5I), we found that GFAP was expressed under both control and neural differentiation conditions. Under differentiation conditions, MAP2 expression was positive compared to control conditions (Figure 5H, I).

\section{Chondrogenesis}

To confirm their chondrogenic differentiation ability, cAMMSCs were cultured with the chondrogenic differentiation medium. The cells were seeded into $15 \mathrm{~mL}$ polypropylene tubes and centrifuged to from a pellet. The cell pellets were incubated with the differentiation medium for 3 weeks. We found that the pellets formed in the bottom of polypropylene tube had an ovoid shape and an opaque body (Figure 6A). Toluidine blue staining was performed to identify chondrogenesis. After differentiation, the pellet showed positive toluidine blue staining (Figure 6B). We also measured the expression patterns of genes associated with chondrogenic markers, such as Aggrecan and COL2A1 via RTPCR (Figure 6C) and quantitiative RT-PCR (Figure 6D). Chondrogenic markers were increased under differentiation conditions, compared to control conditions (Figure 6C, D).

\section{Discussion}

The placenta consists of tissue both maternal and fetal origin after implantation. The placenta consists of fetal placenta from the fetus and uterine placenta from the mother, which is called placentation. The placenta is comprised three-layer structure of the amnion, chorion and decidua. The roles of the placenta are to provide nutrients and oxygen, which are essential for fetal survival and development. The amnion is a thin, nonvascular membrane, which has a two-layer structure: an epithelial monolayer and a stromal layer [18]. In particular, the amnion is the sac that binds the fetus and constructs the environment. The parturient placenta was previously discarded and classified as medical wastes, however, the amniotic membrane has clinical applications in covering wounds and burn lesions and ocular surface reconstruction [7]. In humans, some reports have demonstrated the isolation and characterization of mesenchymal stem cells from whole placenta and amniotic membrane [7-9]. Significantly, they have reported the identification of epithelial stem cells from the amniotic membrane [19]. These isolated cells have the characteristics of stem cells, including, self-renewal and multi-lineage differentiation abilities. Placental derived-stem cells are useful tools for regenerative medicine. They have advantages in cell applications, including the immune privilege, the lack of ethical issues and non-invasive procedures to obtain the amnion. Recently, canine stem cells have been studied for use in cell therapy [20,21]. However, they have been confined to limited stem cell sources. Most of the cells were isolated from canine adiposederived tissue, umbilical cord blood or bone marrow [13,22,23]. Therefore, an established diversity of stem cell source is needed.

In our study, we isolated and cultured cells from 6 different canine amniotic membrane samples (the rate of success was $100 \%$ ). All isolated cells (from 6 samples) showed a very similar cell morphology and ability to be subcultured. We randomly selected three cell lines for characterization (line2 and line 3 were described in supporting data; Figure S1, S2, S3, S4, and S5 and Table S1). All experiments, CPDL, FACS analysis and differentiation studies, were conducted by only the selected cell line (in triplicate).

In our study, we showed the isolation and characterization of MSCs from canine amniotic membranes. Also, Uranio et al. [24] published the study for isolation of canine MSCs from amniotic membranes. However, the article has lack of detailed isolation method and important stem cell characteristics, such as long-term cell maintenance, the immunophenotype, the comprehensive confirmation in differentiation studies. These researches are important for isolation \& characterization of stem cells and veterinary medicine. 

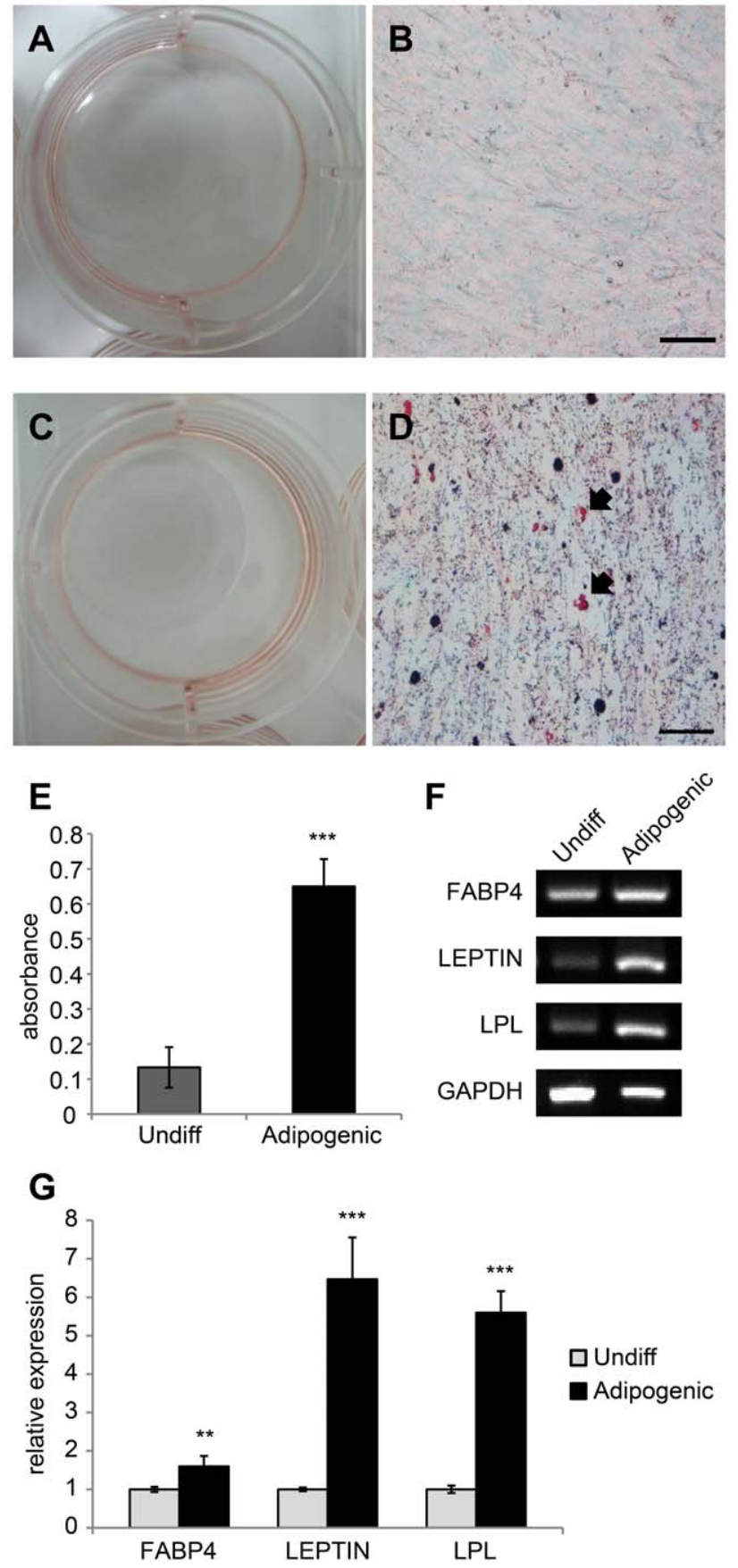

Figure 3. Adipogenic differentiation of CAM-MSCs. (A-D) Oil Red $O$ staining after 3 weeks of adipogenic induction. (A, B) Control cells were grown in the basal culture medium. There was no staining with Oil Red O. (C, D) To assess adipogenic differentiation, the cells were treated with adipogenic induction medium. Fat droplets in differentiated cells were stained by Oil Red O. The black arrow indicates a stained red fat droplet. Scale bar $=50 \mu \mathrm{m}$. For quantification, the stain was solubilized with $100 \%$ isopropanol, and absorbance was measured spectrophotometirically at $500 \mathrm{~nm}$ for $0.5 \mathrm{sec}(\mathrm{E})$. Differentiated cells showed 5 fold greater values than control cells. We performed all these analyses in triplicate. $(F, G)$ Gene expression levels were measured by RT-PCR (F) and quantitative RT-PCR (G) for adipogenic specific markers: FABP4, LEPTIN and LPL. GAPDH was used as a reference for evaluating the quality of mRNA. We performed all these analyses in triplicate and the mean $+/-$ the standard deviation plotted $\left({ }^{* *} ; p<0.01,{ }^{* * *}\right.$; $\mathrm{p}<0.001)$.

doi:10.1371/journal.pone.0044693.g003
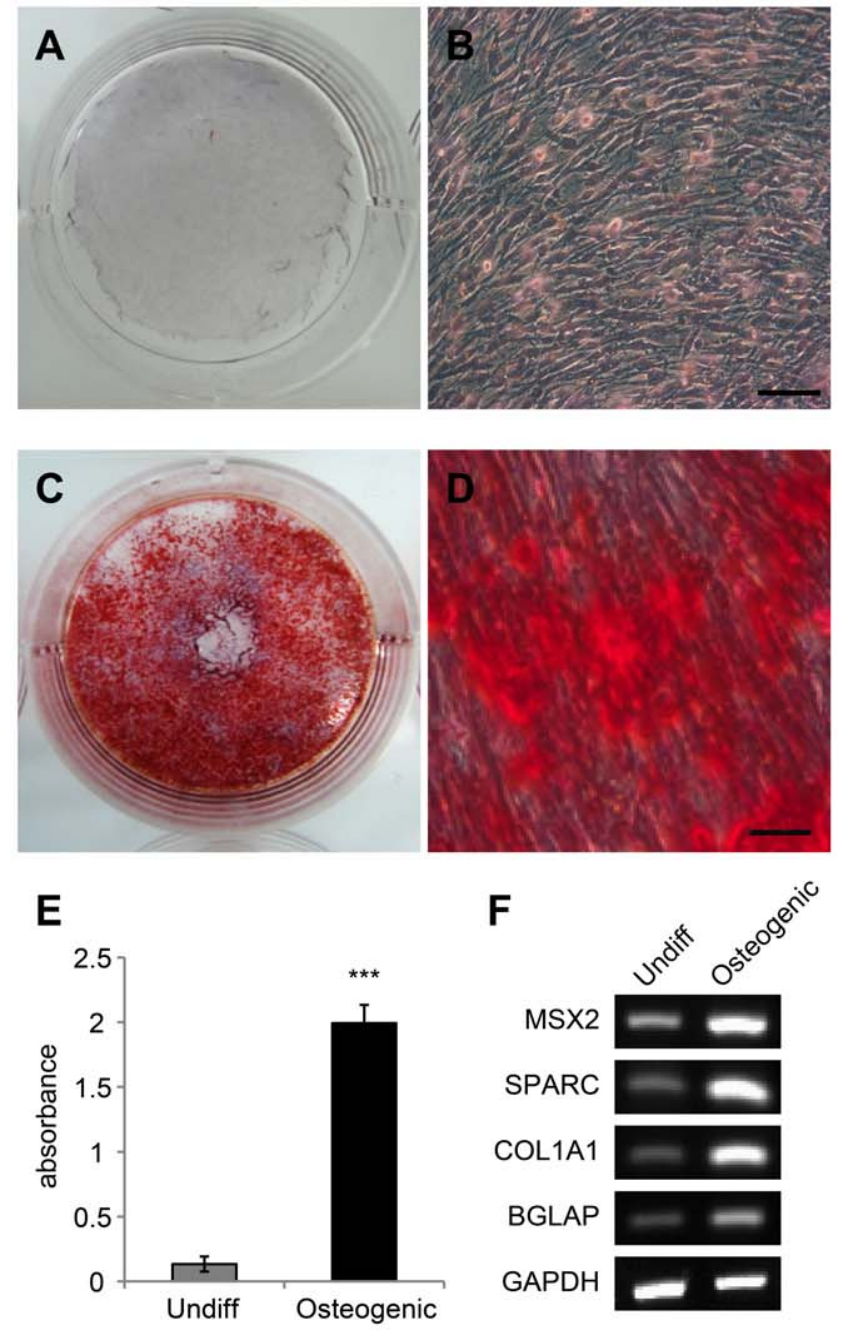

G

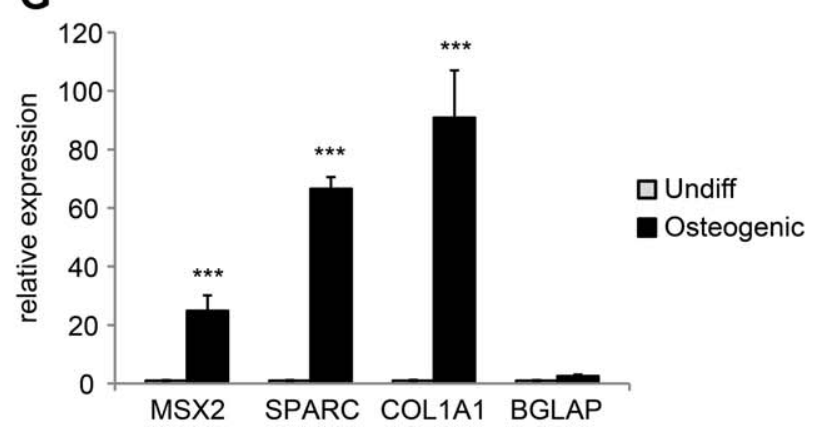

Figure 4. Osteogenic differentiation of CAM -MSCs. (A-D) Cultured were stained with Alizarin Red $S$ after 3 weeks to detect osteogenic induction. (A, B) Control cells were grown in basal culture medium. No staining with Alizarin Red S was observed. (C, D) Cells grown in osteogenic induction medium stained strongly with Alizarin Red S, compared to control cells. Scale bar $=50 \mu \mathrm{m}$. For quantification, stains were solubilized with $100 \mathrm{mM}$ cetylpyridinium chloride, and the absorbance was measured spectrophotometrically at $570 \mathrm{~nm}$ for $0.5 \mathrm{sec}$ (E). Differentiated cells showed 15 -fold greater values than control cells. We performed all these analyses in triplicate. $(F, G)$ Gene expression levels were measured by RT-PCR $(F)$ and quantitative RT-PCR (G) for osteogenic specific markers: MSX2, SPARC, COL1A1 and BGLAP. GAPDH was used as a reference for evaluating the quality of mRNA. We performed all these analyses in triplicate and the mean $+/-$ the standard deviation plotted (***; $\mathrm{p}<0.001)$. doi:10.1371/journal.pone.0044693.g004 

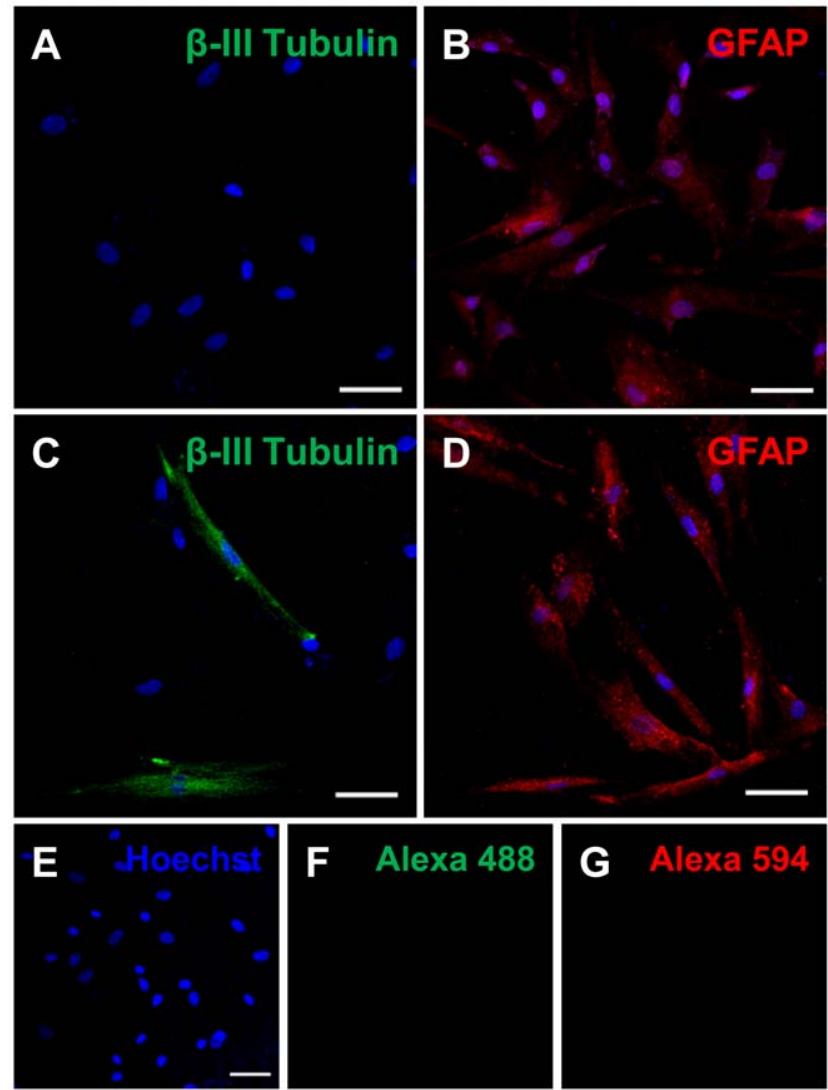

H
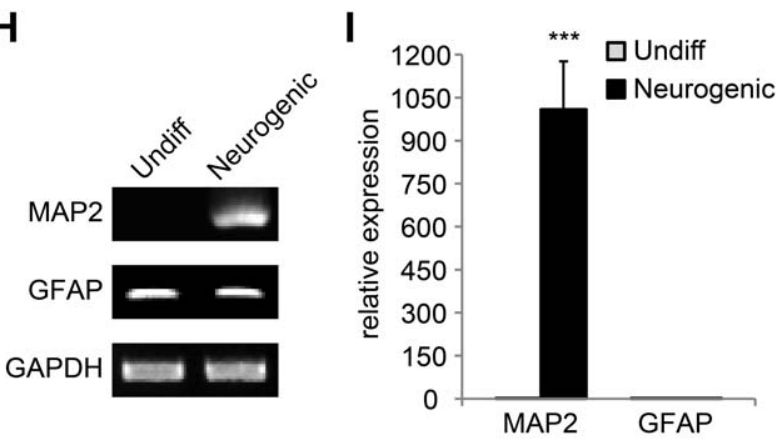

Figure 5. Neural differentiation of CAM-MSCs. The cells were immunostained with GFAP and beta III tubulin, neural specific markers. (A, B) Control cells were cultured with the basal culture medium. Control cells were positive for GFAP but not for beta III tubulin. (C, D) After neural differentiation, cells were stained with GFAP and beta III tubulin. Scale bar $=50 \mu \mathrm{m}$. (E-G) Negative controls were performed only with Alexa 488 (green, F), Alexa 594(red, G) and Hoechst staining for nuclei detection (blue, E). $(\mathrm{H}, \mathrm{I})$ Gene expression levels were measured by RT-PCR $(\mathrm{H})$ and quantitative RT-PCR (I) for neural specific markers: MAP2 and GFAP. GAPDH was used as a reference for evaluating the quality of mRNA. We performed all these analyses in triplicate and the mean $+/-$ the standard deviation plotted (***; $\mathrm{p}<0.001)$.

doi:10.1371/journal.pone.0044693.g005

The amniotic membrane was collected from the subjacent chorion by mechanical detachment. The amniotic membrane was digested with an enzyme treatment for cell isolation. After digestion, cAM-MSCs were grown in basal cultured medium (LG-DMEM with $10 \%$ FBS) for 20 passages. To identify the characteristics of stem cells, we assayed their self-renewal and differentiation abilities. The cell proliferation rate of cAM-MSCs
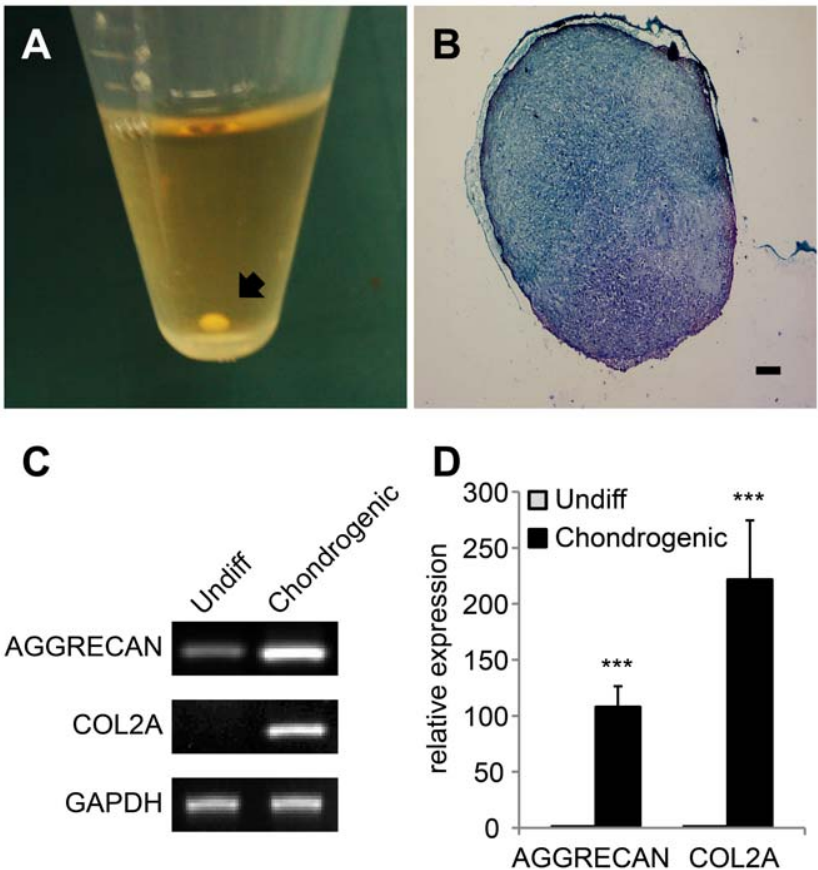

Figure 6. Chondrogenic differentiation of CAM-MSCs. After 3 weeks of chondrogenic induction, pellet formation was observed. (A) Image of an ovoid-shaped chondrogenic pellet. The pellet was formed at the bottom of a $15 \mathrm{ml}$ polypropylene tube. The black arrow indicates a pellet. (B) Toluidine blue staining of chondrogenic pellets. The pellets were embedded in paraffin and cut into $3 \mu \mathrm{m}$ slices, which were mounted on slides. Slides were stained with toluidine blue. The stained tissue showed a typical, cartilaginous tissue phenotype. Scale bar $=100 \mu \mathrm{m}$. (C, D) Gene expression levels were measured by RT-PCR (C) and quantitative RT-PCR (D) for chondrogenic specific markers AGGRECAN and COL2A. GAPDH was used as a reference for evaluating the quality of mRNA. We performed all these analyses in triplicate and the mean $+/-$ the standard deviation plotted $(* * * p<0.001)$. doi:10.1371/journal.pone.0044693.g006

was evaluated via the calculation of cumulative population doubling levels from passage 3 to 20. The stem cell markers were confirmed in passage dependant manner. The morphology of cAM-MSCs was similar to that of typical MSCs with a spindle, fibroblast-like-shape. They also displayed adherence to the plastic culture surface [25]. Immunophenotyping of cAM-MSCs was performed by FACS analysis. The cells expressed the mesenchymal stem cells markers CD90 and CD105, but not hematopoietic surface markers such as CD34 and CD45. The expression pattern of markers indicated that cAM-MSCs have an expression pattern that is characteristic of MSCs [22]. Immnuophenotype characterization indicated that cAM-MSCs are positive for the mesenchymal stem cells markers, CD90 (99.94\%) and CD105 (100\%). In hematopoietic surface makers, there showed negative or some expression pattern for CD45 (1.15\%) and CD34 (0.49\%). In the case of MSCs, usually, CD34 has a negative expression, but Viera et al. [22] and Oh et al. [26] showed that CD34-positive cells (7$10 \%)$ in canine adipose-derived mesenchymal stem cells are more positive population than ours. However, in case of other stem sources, such as bone marrow, amniotic fluid, amnion and umbilical cord matrix, showed negative population of CD34 in MSCs [24,27]. In this regard, we expect that the population of GD34 cells has some differences from origins of stem cells in canine.

We observed the multipotent differentiation ability of cAMMSCs under various induction conditions. We carried out 
differentiation studies of osteogenesis, adipogenesis, neurogenesis and chondrogenesis. In the adipogenesis differentiation sutdy, we confirmed that cAM-MSCs displayed fatty droplets, which were positively stained with Oil Red $\mathrm{O}$ under adipogenic induction conditions. We then eluted the staining in order to quantify differentiation levels, compared to control conditions. By measuring gene expression levels, we showed that adipogenic associated markers were increased after the induction of adipogenic differentiation. After induction of osteogenic differentiation, cAM-MSGs were positively stained with Alizarin Red S. The level of staining was quantified by elution. We showed that the expression levels of osteogenic marker genes, were increased after differentiation. In the neurogenesis differentiation study, we measured the expression of neural-associated markers by immunostaining and RT-PCR. cAM-MSGs express GFAP, as shown by the measurement of both protein and gene levels, even under basal non-differentiating conditions. We confirmed that neural specific markers beta III tubulin and MAP2 were expressed under differentiation conditions. We also found that cAM-MSCs could be differentiated into chondrogenic lineage cells. We confirmed that cAM-MSCs formed pellets, which were positive for toluidine Blue staining under chondrogenic induction medium conditions. Our RT-PCR results showed increased gene levels of markers associated with chondrogenesis.

Taken together, these results show that we have isolated stem cells from amniotic membrane, which have both the characteristics of MSCs and self-renewal ability. Therefore, cAM-MSCs could be a useful source of stem cells for the research of stem cells in canine. Additionally, we suggest that cAM-MSCs have potential therapeutic properties for use in regenerative medicine.

\section{Supporting Information}

Figure S1 Primary culture and the evaluation of CPDL. (A, B) Harvesting of canine amniotic membrane tissue. (C, D) Phase contrast images of cAM-MSCs. Scale bar $=50 \mu \mathrm{m}$. (E) Measuring CPDL of cAM-MSCs. CPDL was evaluated with the formula described in the Materials and Methods section. The CPDL was measured from passage 3 to 20. Cells grew consistently until passage 20 .

(JPG)

Figure S2 Adipogenic differentiation. (A-D) Oil Red O staining after 3 weeks of adipogenic induction. (A, C) Control cells were grown in the basal culture medium. There was no staining

\section{References}

1. Friedenstein AJ Chailakhyan RK, Gerasimov UV (1987) Bone marrow osteogenic stem cells: in vitro cultivation and transplantation in diffusion chambers. Cell Tissue Kinet 20: 263-272.

2. Perin L, Giuliani S, Jin D, Sedrakyan S, Carraro G, et al. (2007) Renal differentiation of amniotic fluid stem cells. Cell Prolif 40: 936-948.

3. Rada T, Reis RL, Gomes ME (2011) Distinct Stem Cells Subpopulations Isolated from Human Adipose Tissue Exhibit Different Chondrogenic and Osteogenic Differentiation Potential. Stem Cell Reviews and Reports 7: 64-76.

4. Lee OK, Kuo TK, Chen WM, Lee KD, Hsieh SL, et al. (2004) Isolation of multipotent mesenchymal stem cells from umbilical cord blood. Blood 103: 1669-1675.

5. Wang JF, Wang LJ, Wu YF, Xiang Y, Xie CG, et al. (2004) Mesenchymal stem/ progenitor cells in human umbilical cord blood as support for ex vivo expansion of CD34(+) hematopoietic stem cells and for chondrogenic differentiation. Haematologica 89: 837-844.

6. Mead LE, Prater D, Yoder MC, Ingram DA (2008) Isolation and characterization of endothelial progenitor cells from human blood. Curr Protoc Stem Cell Biol Chapter 2: Unit 2C 1.

7. Parolini O, Alviano F, Bagnara GP, Bilic G, Buhring HJ, et al. (2008) Concise review: isolation and characterization of cells from human term placenta: outcome of the first international Workshop on Placenta Derived Stem Cells. Stem Cells 26: 300-311. with Oil Red O. (B, D) To assess adipogenic differentiation, the cells were treated with adipogenic induction medium. Fat droplets in differentiated cells were stained by Oil Red O. Scale bar $=50 \mu \mathrm{m}$. (E, F) For quantification, the stain was solubilized with $100 \%$ isopropanol, and absorbance was measured spectrophotometirically at $500 \mathrm{~nm}$ for $0.5 \mathrm{sec}$. We performed all these analyses in triplicate and the mean $+/-$ the standard deviation plotted (***; $\mathrm{p}<0.001)$. (G, H) Gene expression levels were measured by RTPCR.

(JPG)

Figure S3 Osteogenic differentiation. (A-D) The cells were stained with Alizarin Red S after 3 weeks to detect osteogenic induction. (A, C) Control cells were grown in basal culture medium. No staining with Alizarin Red S was observed. (B, D) Cells grown in osteogenic induction medium stained strongly with Alizarin Red S, compared to control cells. Scale bar $=50 \mu \mathrm{m}$. (E, F) For quantification, stains were solubilized with $100 \mathrm{mM}$ cetylpyridinium chloride, and the absorbance was measured spectrophotometrically at $570 \mathrm{~nm}$ for $0.5 \mathrm{sec}$. We performed all these analyses in triplicate and the mean $+/-$ the standard deviation plotted (***; $\mathrm{p}<0.001)$. $(\mathrm{G}, \mathrm{H})$ Gene expression levels were measured by RT-PGR.

(JPG)

Figure S4 Neural differentiation. (A, B) Gene expression levels were measured by RT-PGR.

(JPG)

Figure S5 Ghondrogenic differentiation. (A, D) Image of an ovoid-shaped chondrogenic pellet. The pellet was formed at the bottom of a $15 \mathrm{ml}$ polypropylene tube. (B, E) Toluidine blue staining of chondrogenic pellets. Scale bar $=100 \mu \mathrm{m}$. (C, F) Gene expression levels were measured by RT-PCR.

(JPG)

Table S1 Expression patterns of GD markers with Cell line-2 and Cell line-3. The Values were measured by percentage.

(DOCX)

\section{Author Contributions}

Conceived and designed the experiments: KSK. Performed the experiments: MSS SBP HSK. Analyzed the data: MSS SBP HSK. Contributed reagents/materials/analysis tools: KSK. Wrote the paper: MSS.

8. Diaz-Prado S, Muinos-Lopez E, Hermida-Gomez T, Rendal-Vazquez ME, Fuentes-Boquete I, et al. (2010) Isolation and Characterization of Mesenchymal Stem Cells from Human Amniotic Membrane. Tissue Eng Part C Methods.

9. Kita K, Gauglitz GG, Phan TT, Herndon DN, Jeschke MG (2010) Isolation and characterization of mesenchymal stem cells from the sub-amniotic human umbilical cord lining membrane. Stem Cells Dev 19: 491-502.

10. Mihu CM, Rus Ciuca D, Soritau O, Susman S, Mihu D (2009) Isolation and characterization of mesenchymal stem cells from the amniotic membrane. Rom J Morphol Embryol 50: 73-77.

11. Park SB, Yu KR, Jung JW, Lee SR, Roh KH, et al. (2009) bFGF enhances the IGFs-mediated pluripotent and differentiation potentials in multipotent stem cells. Growth Factors 27: 425-437.

12. Jurga M, Markiewicz I, Sarnowska A, Habich A, Kozlowska H, et al. (2006) Neurogenic potential of human umbilical cord blood: neural-like stem cells depend on previous long-term culture conditions. J Neurosci Res 83: 627-637.

13. Seo MS, Jeong YH, Park JR, Park SB, Rho KH, et al. (2009) Isolation and characterization of canine umbilical cord blood-derived mesenchymal stem cells. J Vet Sci 10: 181-187.

14. Dominici M, Le Blanc K, Mueller I, Slaper-Cortenbach I, Marini F, et al. (2006) Minimal criteria for defining multipotent mesenchymal stromal cells. The International Society for Cellular Therapy position statement. Cytotherapy 8: 315-317. 
15. Masson NM, Currie IS, Terrace JD, Garden OJ, Parks RW, et al. (2006) Hepatic progenitor cells in human fetal liver express the oval cell marker Thy-1. Am J Physiol Gastrointest Liver Physiol 291: G45-54.

16. Nakamura Y, Muguruma Y, Yahata T, Miyatake H, Sakai D, et al. (2006) Expression of CD90 on keratinocyte stem/progenitor cells. Br J Dermatol 154: $1062-1070$.

17. Gargett CE (2006) Identification and characterisation of human endometrial stem/progenitor cells. Aust N Z J Obstet Gynaecol 46: 250-253.

18. Yu SJ, Soncini M, Kaneko Y, Hess DC, Parolini O, et al. (2009) Amnion: a potent graft source for cell therapy in stroke. Cell Transplant 18: 111-118.

19. Miki T, Marongiu F, Dorko K, Ellis EG, Strom SC (2010) Isolation of amniotic epithelial stem cells. Curr Protoc Stem Cell Biol Chapter 1: Unit 1E 3.

20. Byeon YE, Ryu HH, Park SS, Koyama Y, Kikuchi M, et al. (2010) Paracrine effect of canine allogenic umbilical cord blood-derived mesenchymal stromal cells mixed with beta-tricalcium phosphate on bone regeneration in ectopic implantations. Cytotherapy 12: 626-636.

21. Ryu HH, Lim JH, Byeon YE, Park JR, Seo MS, et al (2009) Functional recovery and neural differentiation after transplantation of allogenic adiposederived stem cells in a canine model of acute spinal cord injury. J Vet Sci 10: 273-284.
22. Vieira NM, Brandalise V, Zucconi E, Secco M, Strauss BE, et al. (2010) Isolation, characterization, and differentiation potential of canine adiposederived stem cells. Cell Transplant 19: 279-289.

23. Kamishina H, Deng J, Oji T, Cheeseman JA, Clemmons RM (2006) Expression of neural markers on bone marrow-derived canine mesenchymal stem cells. Am J Vet Res 67: 1921-1928.

24. Filioli Uranio M, Valentini L, Lange-Consiglio A, Caira M, Guaricci AC, et al. (2011) Isolation, proliferation, cytogenetic, and molecular characterization and in vitro differentiation potency of canine stem cells from foetal adnexa: a comparative study of amniotic fluid, amnion, and umbilical cord matrix. Mol Reprod Dev 78: 361-373.

25. Troyer DL, Weiss ML (2008) Wharton's jelly-derived cells are a primitive stromal cell population. Stem Cells 26: 591-599.

26. Oh HJ, Park JE, Kim MJ, Hong SG, Ra JC, et al. (2011) Recloned dogs derived from adipose stem cells of a transgenic cloned beagle. Theriogenology 75: 12211231.

27. Csaki C, Matis U, Mobasheri A, Ye H, Shakibaei M (2007) Chondrogenesis, osteogenesis and adipogenesis of canine mesenchymal stem cells: a biochemical, morphological and ultrastructural study. Histochem Cell Biol 128: 507-520. 